\title{
Regular chromogranin A monitoring facilitated the early detection of a gastrointestinal neuroendocrine tumour in a patient with type 1 diabetes
}

\author{
Zoltan Herold ${ }^{\circledR 1}$, Andrea Uhlyarik ${ }^{(1)}$, Magdolna Herold ${ }^{(1)}$, Peter Nagy ${ }^{\circledR 2}{ }^{2}$, Gergely Denes Huszty ${ }^{3}$, \\ Klara Rosta ${ }^{\circledR 4}{ }^{4}$, Marton Doleschall ${ }^{\circledR 5}$, Aniko Somogyi ${ }^{\circledR 1}$ \\ ${ }^{1}$ Department of Internal Medicine and Haematology, Semmelweis University, Budapest, Hungary \\ ${ }^{2} 1^{\text {st }}$ Department of Pathology and Experimental Cancer Research, Semmelweis University, Budapest, Hungary \\ ${ }^{3}$ Department of Transplantation and Surgery, Semmelweis University, Budapest, Hungary \\ ${ }^{4}$ Department of Obstetrics and Gynaecology, Medical University of Vienna, Vienna, Austria \\ ${ }^{5}$ Molecular Medicine Research Group, Eotvos Lorand Research Network and Semmelweis University, Budapest, Hungary
}

Key words: chromogranin A; diabetes mellitus type 1; enterochromaffin-like cells; atrophic gastritis; neuroendocrine tumours

Neuroendocrine tumours (NET) are rare, slow-growing malignancies in most cases [1]. Chromogranin A (CgA) is used routinely as a biochemical marker to diagnose and monitor NETs. Furthermore, the connection between CgA and diabetes was shown [1,2].

Serum CgA level of type 1 diabetes (T1DM) patients were screened, and the patients with CgA levels above or close to the upper level of normal range (19.4-98.1 $\mathrm{ng} / \mathrm{mL}$ ) were followed up regularly [3]. A 58-year-old male T1DM patient's CgA level was measured at 82.2 $\mathrm{ng} / \mathrm{mL}$ in 2006. The chromogranin A level was slowly rising, and the CgA level was already above the normal range in 2015 (Fig. 1). The increase of CgA was above the expected average annual increase of $\mathrm{CgA}$ [3]. The patient was negative for common CgA-elevating conditions, such as autoimmune gastritis or enterochromaffin-like cell hyperplasia.

In late 2019 the patient's serum CgA level was higher than before, and he started to complain about mild diarrhoea and epigastric discomfort. Due to the high CgA level and uncertain symptoms, further investigations were performed: stool microbiology test (Escherichia coli, Clostridium difficile, Salmonella spp., Shigella spp., Yersinia enterocolitica, Campylobacter spp. and Caliciviridae), gastroscopy, and colonoscopy were all negative; a computed tomography (CT) scan of the abdomen showed a hypervascularised area in the mesentery, caudally from the duodenum. On a previous CT scan dated from 2015, which was also performed due to the elevation in $\mathrm{CgA}$ level, the deviations above were not visible. Fine-needle aspiration of the area indicated on the CT scan confirmed a CgA- and synaptophysin-positive grade I NET with low Ki-67 proliferation rate. Somatostatin receptor scintigraphy was positive for the tumour. Tumour markers, except CgA and 5-hydroxyindoleacetic acid, were within the normal range.

Surgical removal of the tumour and preoperative somatostatin analogue treatment was recommended by the Neuroendocrine Oncology Team, Semmelweis University. The primary tumour $(1.5 \times 1 \mathrm{~cm})$ was resected from the ileum, approximately $50-70 \mathrm{~cm}$ away from the ileocecal valve. A $10 \times 6 \times 5 \mathrm{~cm}$ and a $7 \times 5 \times 4 \mathrm{~cm}$ lymph node conglomerate were also removed from the mesenteric root. Histological findings strengthened the fine-needle aspiration results: tumour was a grade I, pT4N1M0 neuroendocrine lesion with immunohistologically confirmed CgA positivity, and Ki-67 positivity was less than $1 \%$. Eight of the 22 local lymph nodes were positive for metastasis. Based on the evaluation of the oncology team, the tumour had favourable histological prognostic parameters, and there was no sign of distant metastasis; therefore, close monitoring of the patient in every three months using CgA and 5-hydroxyindoleacetic acid measurements was considered sufficient for follow-up. One month after tumour removal, serum CgA returned almost to normal range (103 ng/mL, Fig. 1).

Serum CgA level is a useful marker to monitor the progression of both premalignant lesions and NETs 


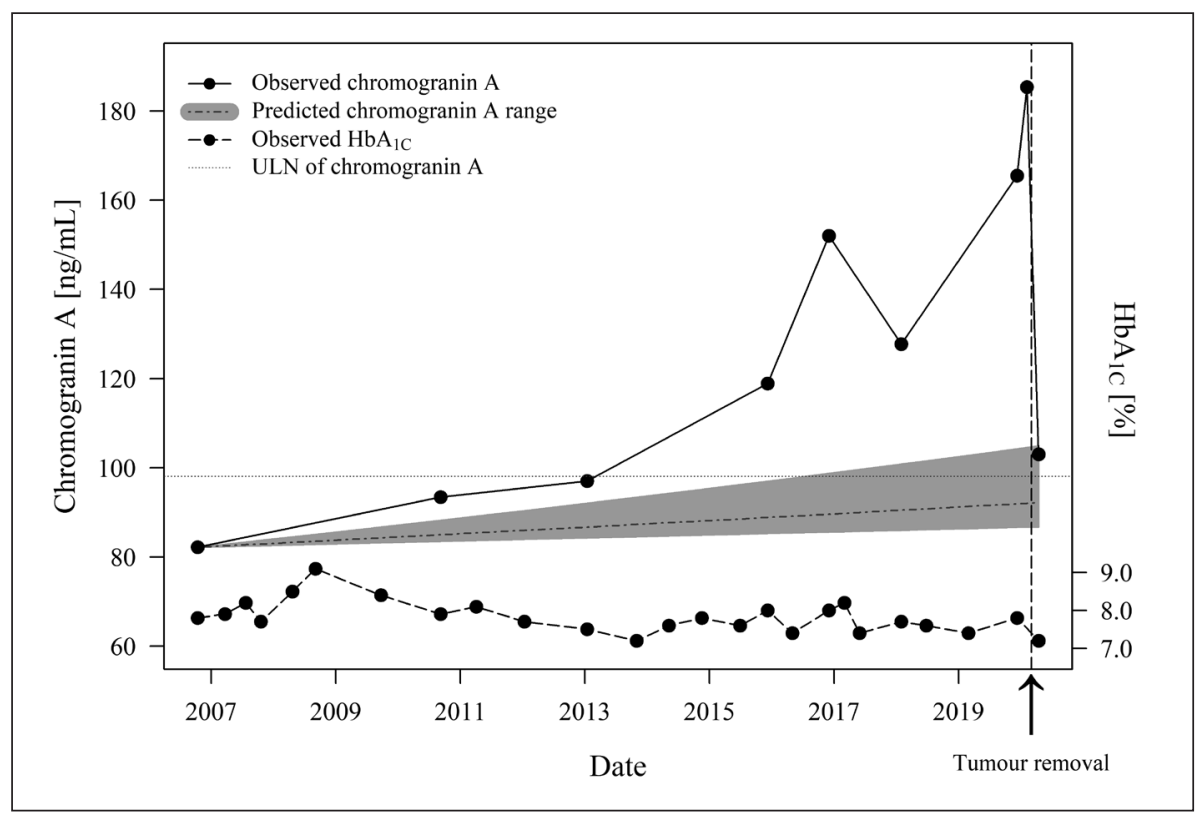

Figure 1. Changes of serum chromogranin A (CgA) levels of the patient between 2006 and 2020. Solid line shows the observed CgA levels, dot-dashed line with the grey range represents the $C g A$ changes predicted with a random intercept mixed effect model [3]. Dotted line is the upper level of normal (ULN) of CgA $(98.1 \mathrm{ng} / \mathrm{mL})$, and the dashed line at the bottom shows glycated haemoglobin $\left(\mathrm{Hb}_{1 \mathrm{c}}\right)$ levels during the $\sim 14$-year period

$[1,3,4]$. The causal relationship between the high and increasing levels of serum CgA and the presence of enterochromaffin-like cell hyperplasia, autoimmune gastritis, and NETs in patients with T1DM is highly probable [3, 4]. The removal of a gastrointestinal NET from this patient drastically decreased the serum CgA level supporting the described causal relationship. This case has clearly proven the usefulness of $\mathrm{CgA}$ as a marker to detect and follow-up NETs in patients with T1DM. During approximately 15 years of our observation this was the third, but best "CgA-documented" NET discovered from 200 T1DM patients in our outpatient clinic. NETs have a higher incidence rate in T1DM than in the general population [5]; therefore, the routine measurement of CgA in T1DM (annually or every two years, for example) facilitates the early detection of gastrointestinal NETs. Even a moderate increase in the level of serum CgA may draw the clinicians' attention, and further examinations by imaging techniques can be initiated, as well as early intervention. We propose that serum CgA in patients with T1DM serves as a useful biomarker for early detection of gastrointestinal NETs.

\section{Information on grants and other funding sources} Z.H. was supported by Wörwag Pharma Kft (Wörwag Research Prize for PhD students). MD was supported by the Hungarian Academy of Sciences (Janos Bolyai Research Scholarship) and the Ministry for Innovation and Technology (UNKP-19-4 New National Excellence
Program). Research was supported by the National Research, Development, and Innovation Office (grant number K-116128) and by the Hungarian Diabetes Association.

\section{Author disclosures}

Authors declare no conflict of interests.

\section{Author contributions}

All authors contributed to the design of the work. Z.H., M.D., and M.H. drafted the manuscript. Z.H. and M.H. prepared data, performed statistical modelling, and drew Figure 1. All authors critically revised the manuscript. All authors approved the final version. M.D. and A.S. share senior authorship.

\section{References}

1. Wang R, Zheng-Pywell R, Chen HA, et al. Management of Gastrointestinal Neuroendocrine Tumors. Clin Med Insights Endocrinol Diabetes. 2019; 12: 1179551419884058, doi: 10.1177/1179551419884058, indexed in Pubmed: 31695546.

2. Herold Z, Doleschall M, Kovesdi A, et al. Chromogranin A and its role in the pathogenesis of diabetes mellitus. Endokrynol Pol. 2018; 69(5): 598-610, doi: 10.5603/EP.a2018.0052, indexed in Pubmed: 30074235.

3. Herold Z, Herold M, Nagy P, et al. Serum chromogranin A level continuously rises with the progression of type 1 diabetes, and indicates the presence of both enterochromaffin-like cell hyperplasia and autoimmune gastritis. J Diabetes Investig. 2019 [Epub ahead of print], doi: 10.1111/jdi.13203, indexed in Pubmed: 31883432.

4. De Block CEM, Colpin G, Thielemans K, et al. Neuroendocrine tumor markers and enterochromaffin-like cell hyper/dysplasia in type 1 diabetes. Diabetes Care. 2004; 27(6): 1387-1393, doi: 10.2337/diacare.27.6.1387, indexed in Pubmed: 15161793.

5. Bizzaro N, Antico A, Villalta D. Autoimmunity and Gastric Cancer. Int J Mol Sci. 2018; 19(2), doi: 10.3390/ijms19020377, indexed in Pubmed: 29373557 